\title{
MRI Features Can Predict 1p/19q Status in Intracranial Gliomas
}

\author{
(D)A. Lasocki, (D)F. Gaillard, (D)A. Gorelik, and (D) M. Gonzales
}

\begin{abstract}
BACKGROUND AND PURPOSE: The 2016 revision of the World Health Organization Classification of Tumors of the Central Nervous System mandates codeletion of chromosomes $1 p$ and $19 q$ for the diagnosis of oligodendroglioma. We studied whether conventional MR imaging features could predict $1 \mathrm{p} / 19 \mathrm{q}$ status.
\end{abstract}

MATERIALS AND METHODS: Patients with previous 1p/19q testing were identified through pathology department records, typically performed on the basis of an oligodendroglial component on routine histology; 69 patients met the inclusion criteria. Preoperative imaging of patients with grade II or III gliomas was retrospectively assessed by 2 neuroradiologists, blinded to the 1p/19q status. Thirteen MR imaging features were first assessed in a small initial cohort $(n=10)$, after which the criteria were narrowed for the remaining patients as a validation cohort.

RESULTS: There was $85 \%$ agreement between radiologists for the overall prediction of $1 \mathrm{p} / 19 \mathrm{q}$ status in the validation cohort, with an accuracy of $84 \%$. The presence of $>50 \%$ T2-FLAIR mismatch and calcification was found to be the most useful for predicting $1 \mathrm{p} / 19 \mathrm{q}$ status. The $>50 \%$ T2-FLAIR mismatch variable was demonstrated in 14 tumors and had 100\% specificity for identifying a noncodeleted tumor $(P=$ .001 ), with $97 \%$ interobserver correlation. Calcification was visualized in 7 tumors, 6 of which were 1p/19q codeleted (specificity, $97 \% ; P=$ .006), with $100 \%$ interobserver correlation.

CONCLUSIONS: The presence of $>50 \%$ T2-FLAIR mismatch is highly predictive of a noncodeleted tumor, while calcifications suggest a $1 \mathrm{p} / 19 \mathrm{q}$ codeleted tumor. If formal $1 \mathrm{p} / 19 \mathrm{q}$ testing is not possible, a combined MR imaging-histologic assessment may improve the diagnostic accuracy over histology alone.

ABBREVIATIONS: TCGA/TCIA = The Cancer Genome Atlas/The Cancer Imaging Archive; WHO = World Health Organization

$\mathrm{T}$ he diagnostic criteria for astrocytic and oligodendroglial tumors have recently been updated by the World Health Organization (WHO), leading to a greater reliance on molecular markers over just the histologic phenotype. ${ }^{1}$ An important update has been the mandating of codeletion of chromosomes $1 \mathrm{p}$ and $19 \mathrm{q}$ for the diagnosis of oligodendroglioma, in addition to the presence of a mutation in the isocitrate dehydrogenase (IDH) genes, either type 1 (IDH1) or type 2 (IDH2). ${ }^{1}$ Thus, the previous heterogeneous

Received January 31, 2017; accepted after revision December 29.

From the Department of Cancer Imaging (A.L.), Peter MacCallum Cancer Centre Melbourne, Victoria, Australia; Department of Radiology (A.L., F.G.), Melbourne EpiCentre (A.G.), and Department of Anatomical Pathology (M.G.), The Royal Melbourne Hospital, Parkville, Victoria, Australia; Monash Imaging (A.L.), Monash Health, Clayton, Victoria, Australia; and Departments of Medicine (A.G.) and Pathology (M.G.), The University of Melbourne, Parkville, Victoria, Australia.

Please address correspondence to Arian Lasocki, MD, Department of Cancer Imaging, Peter MacCallum Cancer Centre, Grattan St, Melbourne, Victoria, Australia 3000; e-mail: arian.lasocki@petermac.org

http://dx.doi.org/10.3174/ajnr.A5572 group of oligoastrocytomas, containing both neoplastic astrocytic and oligodendroglial components, has been largely abolished, with true mixed oligoastrocytomas now being rare. ${ }^{1}$ As the number and importance of distinct genetic mutations increases, however, there is the potential for a substantial increase in cost. MR imaging has the potential to improve the targeting of molecular testing to those patients with a greater likelihood of a positive result. ${ }^{2}$ In addition, $1 \mathrm{p} / 19 \mathrm{q}$ testing is not readily available in many centers, and there may be insufficient tissue to perform $1 \mathrm{p} / 19 \mathrm{q}$ testing in some cases. It is not clear how to optimize diagnosis in this context. In such situations, the WHO recommends labeling the tumor on the basis of its histologic phenotype, but with the suffix "NOS" (not otherwise specified). ${ }^{1}$ This labeling essentially reverts to the prior classification, the deficiencies of which have been acknowledged. ${ }^{1}$

A conventional MR imaging feature labeled "T2-FLAIR mismatch" has been studied by Patel et $\mathrm{al}^{3}$ in patients from The Cancer Genome Atlas/The Cancer Imaging Archive (TCGA/TCIA) 
cohort. This feature was found in 15 of 125 cases of lower grade gliomas, all of which were $1 \mathrm{p} / 19 \mathrm{q}$ noncodeleted. The authors subsequently validated their findings in 82 patients from their own institution, with all 10 tumors having this appearance being $1 \mathrm{p} /$ 19q noncodeleted. ${ }^{3}$ T2-FLAIR mismatch is equivalent to the MR imaging appearances of so-called "protoplasmic" astrocytomas; these tumors typically demonstrate a component with high signal on T2WI and substantial suppression on T2-FLAIR imaging. ${ }^{4}$ This T2-FLAIR suppression was found in all 8 protoplasmic astrocytomas in the series by Tay et $\mathrm{al}^{4}$ and occupied more than half of the tumor in 5 of their 8 cases. Although protoplasmic astrocytomas are no longer recognized as a distinct entity in the updated WHO classification, now being included within the diagnosis of diffuse astrocytoma, ${ }^{1}$ this characteristic appearance provides an explanation for the correlation between T2-FLAIR mismatch and $1 \mathrm{p} / 19 \mathrm{q}$ noncodeleted tumors.

In contrast, the presence of calcification on CT has been found to predict $1 \mathrm{p} / 19 \mathrm{q}$ loss of heterozygosity. ${ }^{5}$ Noncircumscribed borders have also been shown to correlate with $1 \mathrm{p} / 19 \mathrm{q}$ codeletion, occurring in $92 \%$ of molecular oligodendrogliomas in a recent series by Johnson et al, ${ }^{6}$ but this appearance is not specific because it was also present in $45 \%$ of noncodeleted tumors. Other conventional imaging features suggested as being typical of oligodendrogliomas include a cortical-subcortical location, ${ }^{7}$ though it is not clear whether this remains true in the current molecular era. Also before the classification update, elevated CBV was a wellknown feature of oligodendrogliomas related to their "chicken wire" vascularity. ${ }^{8} \mathrm{CBV}$ elevation has been associated with chromosome $1 \mathrm{p}$ deletion, albeit in a small cohort. ${ }^{9}$ The value of CBV elevation for the differentiation between codeleted and noncodeleted tumors is limited, however, by the association with highgrade gliomas. ${ }^{10}$ This limitation is of particular relevance to anaplastic gliomas but also relevant to WHO grade II tumors, due to the risk of sampling error. ${ }^{11}$

The purpose of this study was to determine whether conventional MR imaging features can be used to predict $1 \mathrm{p} / 19 \mathrm{q}$ status in WHO grade II and III gliomas.

\section{MATERIALS AND METHODS \\ Patient Selection}

Institutional human research ethics committee approval was obtained. Patients with previous $1 \mathrm{p} / 19 \mathrm{q}$ testing were identified through the records of the Department of Anatomical Pathology at The Royal Melbourne Hospital. Testing for $1 p / 19 q$ by fluorescence in situ hybridization had been performed on samples received between August 2010 and August 2016 as previously described, ${ }^{12}$ assessing 20 nuclei at 10 different sites for a total of 200 nuclei. Reference to a test signal ratio of $<0.8$ was regarded as indicating detection. We included WHO grade II or III gliomas with the following histologic diagnoses: astrocytoma, oligodendroglioma, oligoastrocytoma, or diffuse glioma. Testing for $1 \mathrm{p} /$ $19 q$ had generally been performed due to the presence of an oligodendroglial component or diagnostic uncertainty on standard histologic assessment, though testing was performed more broadly in the last few months of the study cohort (thus in a minority of patients) as a result of the updated WHO criteria. IDH1, ATRX, and TP53 immunohistochemistry was also per-

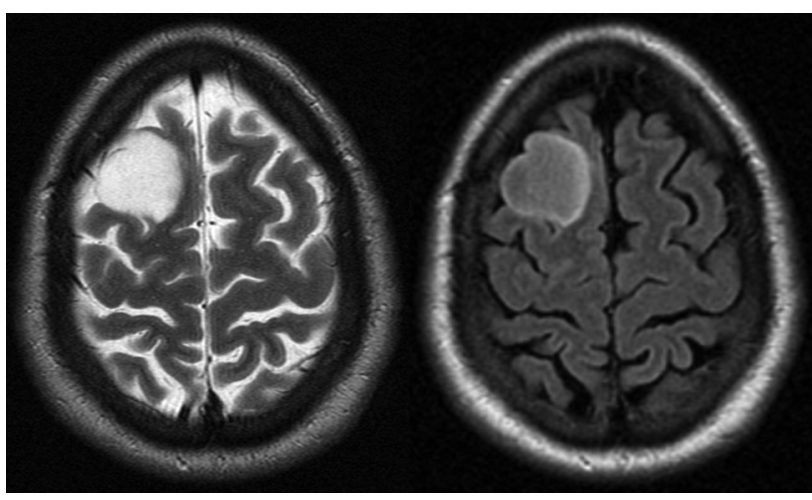

FIG 1. Axial T2 and FLAIR images of a patient with a low-grade right frontal lobe glioma. It is markedly T2-hyperintense, like CSF, with the majority of the tumor demonstrating substantially lower signal on the FLAIR sequence. This tumor was $1 \mathrm{p} / 19 \mathrm{q}$ noncodeleted.

formed for all patients, but more definitive IDH mutation testing was not routinely available for patients with immunohistochemistry negative for $I D H 1$. Histologic assessment was performed by subspecialist neuropathologists for all patients. Patients were excluded if they had a histologic diagnosis other than those listed, a WHO grade IV tumor, or no preoperative MR imaging available.

\section{MR Imaging Assessment}

Preoperative MRIs were reviewed by 2 neuroradiologists with a subspecialty interest in neuro-oncology. MRIs were performed on several different scanners at our institution ( 35 at $1.5 \mathrm{~T}$ and 12 at $3 \mathrm{~T}$ ) or incorporated from a variety of outside institutions $(n=$ 22). Assessment was performed in 2 stages. Initially, 10 cases thought by the study neuropathologist to be histologically characteristic of each entity (five $1 \mathrm{p} / 19 \mathrm{q}$ codeleted and 5 noncodeleted tumors, all WHO grade II) were reviewed, assessing a larger number of conventional MR imaging features based on the Visually Accessible Rembrandt Images (VASARI) feature set. ${ }^{13}$ These features were the following: lobar location; tumor laterality; cortical involvement (the percentage of the total perimeter of the tumor within the cortex); enhancement quality; proportions of enhancing tumor, non-contrast-enhancing tumor, necrosis, and edema; proportion of T2-FLAIR mismatch; cysts; T1/FLAIR ratio; definition of non-contrast-enhancing tumor margin; and calcification. Proportions were stratified as $0 \%, 1 \%-5 \%, 6 \%-33 \%, 34 \%-67 \%$, $68 \%-95 \%$, or $>95 \%$, as per the VASARI criteria. ${ }^{13}$ Calcification was only considered present if it could be confidently differentiated from hemorrhage, either by CT or the phase component of susceptibility-weighted imaging when performed. T2-FLAIR mismatch was subjectively determined as the proportion of the tumor demonstrating high signal on T2WI and substantial suppression on T2-FLAIR imaging (Fig 1). Finally, an overall subjective MR imaging prediction of $1 \mathrm{p} / 19 \mathrm{q}$ status was made on the basis of the combination of findings because at this initial stage of the evaluation, the relative specificities of each feature were not clear.

After the initial cohort of 10 patients, the cases were discussed and the imaging features included in the subsequent assessment were rationalized to those thought to be most helpful in suggesting either a $1 \mathrm{p} / 19 \mathrm{q}$ codeleted or noncodeleted tumor, to minimize the chances of finding an association by chance alone. These fea- 
tures were the presence of calcification, the extent of T2-FLAIR mismatch, and the extent of cortical involvement. The other MR imaging features were thought to have too much overlap between the 2 tumor types. Calcification was based on CT for most patients (preoperative CT was available for 40 patients and was performed within 48 hours postoperatively in another 15), supplemented by susceptibility-weighted imaging, including phase images, in 12 patients. T2-FLAIR mismatch and cortical involvement were stratified as $<33 \%, 33 \%-50 \%$, or $>50 \%$, to determine the optimal threshold for predicting $1 \mathrm{p} / 19 \mathrm{q}$ status. As before, an overall MR imaging prediction on $1 \mathrm{p} / 19 \mathrm{q}$ status was also made on the basis of the sequential assessment of calcification (its presence indicating a codeleted tumor), T2-FLAIR mismatch ( $>50 \%$ indicating a noncodeleted tumor), and substantial cortical involvement ( $>50 \%$ suggesting a codeleted tumor). If all 3 features were absent, the assessment was then subjective, typically considering $33 \%-50 \%$ T2-FLAIR mismatch or cortical involvement as a positive finding (for a noncodeleted and codeleted tumor, respectively). This assessment was performed for the remaining patients in the cohort.

\section{Statistical Analysis}

$\kappa$ statistics were calculated to determine interobserver agreement between the 2 reviewers, and the Fisher exact test was used to determine the association between $1 \mathrm{p} / 19 \mathrm{q}$ status and the presence of calcification and $>50 \%$ T2-FLAIR mismatch. The analysis was performed by using STATA 12 (StataCorp, College Station, Texas).

\section{RESULTS}

\section{Histology}

Of 92 patients initially identified as having undergone $1 \mathrm{p} / 19 \mathrm{q}$ testing, 23 were excluded ( 14 did not have preoperative MR imaging available for review, 4 had a grade IV tumor, and 5 had other histologic diagnoses), leaving 69 patients, comprising the initial cohort of 10 patients and the 59 patients in the validation cohort. The histologic diagnoses in the validation cohort (based on pathology department records) consisted of oligoastrocytomas ( $n=$ $35)$, oligodendrogliomas $(n=12)$, astrocytomas ( $n=6$, including 1 protoplasmic astrocytoma based on the prior WHO classification), and diffuse gliomas $(n=6)$. The tumors labeled as diffuse gliomas were diagnosed after the release of the 2016 revision of the WHO classification. Forty-three tumors were WHO grade II (73\%); the remaining 16 were grade III. Twenty-one of the 59 tumors $(36 \%)$ in the validation cohort were $1 \mathrm{p} / 19 \mathrm{q}$ codeleted $(12$ phenotypic oligoastrocytomas, 7 oligodendrogliomas, 1 astrocytoma, and 1 diffuse glioma); the remaining 38 tumors were noncodeleted (23 oligoastrocytomas, 5 oligodendrogliomas, 5 astrocytomas, and 5 diffuse gliomas).

Forty-seven patients had immunohistochemistry positive for IDH1. IDH pyrosequencing was also performed for one of the patients with immunohistochemistry negative for $I D H 1$, demonstrating an R132S mutation. Five of the 10 noncodeleted tumors with immunohistochemistry negative for IDH1 demonstrated both ATRX and TP53 mutations: 4 had mutations in either ATRX or TP53, and 1 patient had immunohistochemistry negative for both. All except 1 of the 1p/19q codeleted tumors had immuno- histochemistry positive for IDH1. This tumor had the appearance of an oligodendroglioma on standard histology, with wild-type ATRX and TP3. The tumor was thus suspected of having a non$\mathrm{R} 132 \mathrm{H}$ IDH mutation, given the strong association between $1 \mathrm{p} /$ $19 \mathrm{q}$ codeleted oligodendrogliomas and IDH mutations. ${ }^{14}$

\section{MR Imaging Assessment: Initial 10 Patients}

The 2 radiologists agreed on the diagnosis in 9 of the 10 patients in the initial cohort, with $100 \%$ agreement with $1 \mathrm{p} / 19 \mathrm{q}$ status in these cases. The only discrepancy was a patient with calcification (suggestive of $1 \mathrm{p} / 19 \mathrm{q}$ codeletion) but features otherwise suggestive of a noncodeleted tumor. This tumor had $1 \mathrm{p} / 19 \mathrm{q}$ codeletion.

\section{MR Imaging Assessment: Validation Cohort}

There was good agreement between radiologists for the overall prediction of $1 \mathrm{p} / 19 \mathrm{q}$ status $(\kappa=0.61 ; 95 \% \mathrm{CI}, 0.4-0.8 ; P<.001)$, with both giving the same diagnosis in 50 of the 59 cases $(85 \%)$. Both radiologists had $78 \%$ accuracy in predicting $1 \mathrm{p} / 19 \mathrm{q}$ status, which improved to $84 \%$ for the 50 cases with agreement between radiologists. Interobserver correlation varied among individual MR imaging features. Calcification had 100\% correlation between radiologists. The agreement between radiologists for the T2-FLAIR mismatch variable was almost perfect, with absolute agreement in 57 of 59 patients and 2 with \pm 1 point difference (weighted $\kappa=0.88 ; 95 \%$ CI, $0.76-1.00 ; P<.001$ ). The cortical involvement variable had poor interobserver correlation, however, at $42 \%$, which largely accounted for the differences in the overall MR imaging prediction of $1 \mathrm{p} / 19 \mathrm{q}$ status between the 2 readers.

Calcification was visualized in 7 tumors, 6 of which were $1 \mathrm{p} /$ $19 \mathrm{q}$ codeleted $(P=.006)$. These consisted of 5 oligoastrocytomas, 1 oligodendroglioma, and 1 diffuse glioma based on initial phenotypic assessment. Fourteen tumors demonstrated $>50 \%$ T2FLAIR mismatch according to both readers, all noncodeleted $(P=.001)$. Importantly, the presence of $>50 \%$ T2-FLAIR mismatch correctly identified the noncodeleted status in 2 patients when this was not suspected on initial histology (both being phenotypic oligodendrogliomas). Of the remaining 12, eight were initially labeled as oligoastrocytomas; 3 , as astrocytomas; and 1 , as a diffuse glioma. Of note, the 1 protoplasmic astrocytoma in this cohort did demonstrate $>50 \%$ T2-FLAIR mismatch. Eleven of the 14 tumors with $>50 \%$ T2-FLAIR mismatch had immunohistochemistry positive for $I D H 1$, while the 3 patients with immunohistochemistry negative for IDH1 all demonstrated both ATRX and TP53 mutations, suggesting that they had non-R132H IDH mutations. ${ }^{15}$ Only 1 tumor had 33\%-50\% T2-FLAIR mismatch, also noncodeleted. Greater than 50\% cortical involvement (Fig 2) was identified by both readers in 4 patients, all with $1 \mathrm{p} / 19 \mathrm{q}$ codeletion. Only 1 patient had the presence of $>1$ of these 3 features: This patient had both calcification and $>50 \%$ cortical involvement, both features correctly predicting $1 \mathrm{p} / 19 \mathrm{q}$ codeletion.

Specificity was high for both the $>50 \%$ T2-FLAIR mismatch variable ( $100 \%$ specific for predicting a noncodeleted tumor) and the presence of calcification ( $97 \%$ specific for predicting a codeleted tumor). The positive predictive values were also high at $100 \%$ and $86 \%$. Sensitivity was relatively low, however, at $37 \%$ and $29 \%$, because most tumors (38 of 59) did not have either of 


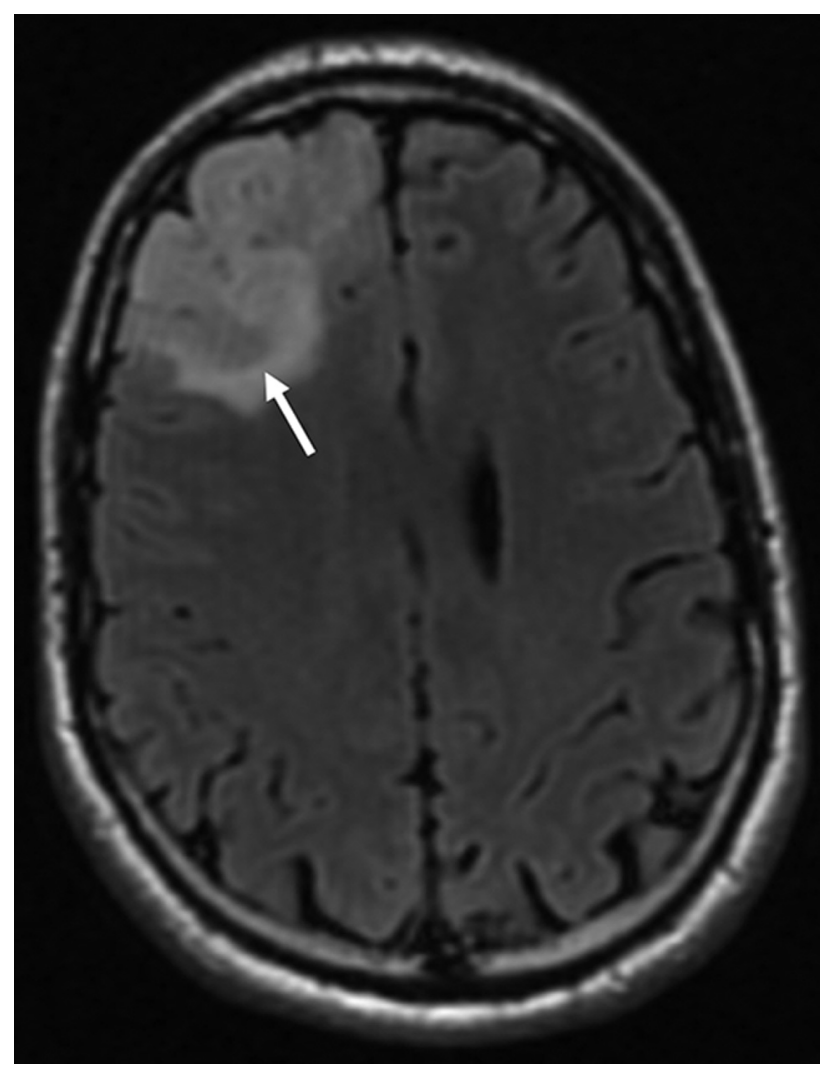

FIG 2. Axial FLAIR image showing a right frontal tumor with $>50 \%$ cortical involvement. The margin of the expanded cortex is demonstrated by the white arrow. This tumor was $1 \mathrm{p} / 19 \mathrm{q}$ codeleted.

these 2 features. Given the poor interobserver correlation for the cortical involvement variable, it was not considered appropriate to determine consensus results; thus, an overall correlation with $1 \mathrm{p} / 19 \mathrm{q}$ status was not calculated. For 1 reader, the overall accuracy was $71 \%$ by using cut-offs of either $>33 \%$ or $>50 \%$ cortical involvement as predictive of $1 \mathrm{p} / 19 \mathrm{q}$ codeletion; for the second reader, the overall accuracy was $61 \%$ and $53 \%$, respectively. With a cutoff of $>33 \%$ cortical involvement (which fared better across the 2 readers), the sensitivity and specificity were $55 \%$ and $81 \%$, respectively, for one reader, and $78 \%$ and $50 \%$ for the other reader.

Using the presence of $>50 \%$ T2-FLAIR mismatch and/or calcification to predict $1 \mathrm{p} / 19 \mathrm{q}$ status in the 35 patients with phenotypic oligoastrocytomas decreased the number of patients with indeterminate $1 \mathrm{p} / 19 \mathrm{q}$ status by 13 (to 22 ) and thus doubled the number of patients in whom $1 \mathrm{p} / 19 \mathrm{q}$ status was predicted correctly (from 13 to 26). If these MR imaging features were used in preference to the histologic phenotype when present (in the 2 phenotypic oligodendrogliomas demonstrating $>50 \%$ T2-FLAIR mismatch, as described above), 1p/19q status was correctly predicted in a further 2 patients, decreasing the number of incorrect predictions from 5 to 3 .

\section{DISCUSSION}

The simple MR imaging assessment described was overall moderately accurate for predicting $1 \mathrm{p} / 19 \mathrm{q}$ status, but the accuracy of $82 \%$ is insufficient to replace formal $1 \mathrm{p} / 19 \mathrm{q}$ testing for all patients. The inaccuracy is partly because, in some tumors, none of

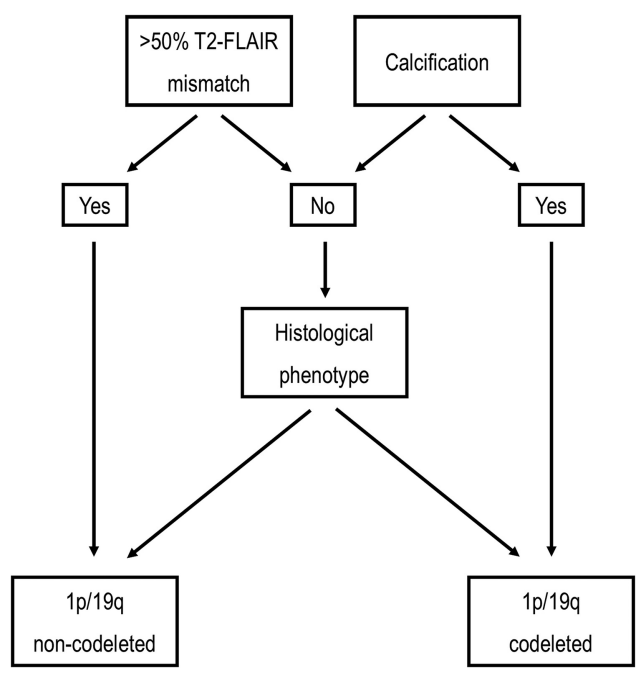

FIG 3. A suggested combined MR imaging-histology method of determining $1 p / 19 q$ status when formal testing is not possible.

the key MR imaging features were present. More important for the clinical setting, however, a substantial proportion of tumors demonstrated MR imaging features that can predict $1 \mathrm{p} / 19 \mathrm{q}$ status with high specificity, including in cases in which this is not expected from the histologic phenotype. Thus, $>50 \%$ T2-FLAIR mismatch is strongly predictive of a noncodeleted tumor, while the tumor is likely $1 \mathrm{p} / 19 \mathrm{q}$-codeleted if there are calcifications. Of these $2 \mathrm{MR}$ imaging features, $>50 \%$ T2-FLAIR mismatch was both more sensitive and more specific than the presence of calcifications. No patients had both features, suggesting that they are mutually exclusive, at least in large part.

Histology remains a crucial first step, both to confirm the diagnosis of glioma (because these MR imaging features can also occur in other tumors and nonneoplastic conditions) and for glioma grading. ${ }^{14}$ Nevertheless, once a grade II or III glioma has been diagnosed (and other differentials have been excluded), MR imaging is very useful for predicting $1 \mathrm{p} 19 \mathrm{q}$ status. The presence of $>50 \%$ T2-FLAIR mismatch or calcification may provide a surrogate marker of $1 \mathrm{p} / 19 \mathrm{q}$ status in cases in which formal testing cannot be performed, for example due to financial or geographic limitations. This feature would be most useful in patients with an indeterminate histologic phenotype, specifically those labeled as "oligoastrocytoma NOS" according to the updated WHO classification. ${ }^{1}$ The MR imaging features may even negate the need for formal $1 \mathrm{p} / 19 \mathrm{q}$ testing, especially if the phenotypic appearances are supportive. Figure 3 illustrates a possible combined MR imaging-histology assessment method for cases in which formal $1 \mathrm{p} /$ 19q testing is not possible. First, the tumor is assessed for the presence of $>50 \%$ T2-FLAIR mismatch or calcifications. If neither of these features is present or if the results are discordant (likely a very rare occurrence), the diagnosis reverts to the histologic phenotype. While there will be rare exceptions to this method, because not all tumors having calcifications are noncodeleted (as demonstrated by 1 patient in our cohort), our results show that this method is likely to be more accurate than using the histologic phenotype alone.

Johnson et $\mathrm{al}^{6}$ have shown that noncircumscribed borders correlate with $1 \mathrm{p} / 19 \mathrm{q}$ codeletion, but given that this appearance 
was also present in $45 \%$ of noncodeleted tumors, this MR imaging feature is not sufficiently specific to predict codeletion with confidence. Rather, to predict $1 \mathrm{p} / 19 \mathrm{q}$ status with high specificity, it may be more useful to use circumscribed borders as predictive of a noncodeleted tumor, albeit with limited sensitivity. The suggested combined assessment method could also potentially be extended to cases in which MR imaging is not available, because CT provides useful information. CT is excellent for the detection of calcifications, while the correlate of T2-FLAIR mismatch on CT is a markedly hypodense tumor. Using these features may also have some value even if formal $1 \mathrm{p} / 19 \mathrm{q}$ testing is available. Both of the most common methods of determining $1 \mathrm{p} / 19 \mathrm{q}$ status (fluorescence in situ hybridization and polymerase chain reactionbased microsatellite loss of heterozygosity) have been shown to occasionally produce false-positive results, ${ }^{16}$ and there is also a small risk of sampling error due to the inherent heterogeneity of glial series tumors. ${ }^{17-19}$ In contrast, MR imaging provides the potential to overcome this limitation due to its ability to assess the entire tumor. ${ }^{11} \mathrm{~A}$ discrepancy between the MR imaging appearances and formal $1 p / 19 q$ testing may thus raise the possibility of sampling error or a mixed tumor.

Dominant cortical involvement was the least useful feature in our cohort due to the high interobserver variability and low incidence. In addition, it is likely to be the least specific feature, especially in smaller tumors, because cortical involvement is also frequently present in astrocytomas. ${ }^{20,21}$ These limitations of the cortical involvement variable account for some of the inaccuracies in the overall prediction of $1 \mathrm{p} / 19 \mathrm{q}$ status. We also assessed the 2 possible thresholds of $>50 \%$ or $>33 \%$ for the proportions of T2-FLAIR mismatch and cortical involvement. This assessment is most relevant to T2-FLAIR mismatch, given the limitations of cortical involvement discussed above. Of the 2 thresholds, we think that $>50 \%$ is the most appropriate. Only 1 tumor had $33 \%-$ $50 \%$ T2-FLAIR mismatch; thus, dropping the threshold to $>33 \%$ would only slightly increase the sensitivity of MR imaging assessment in identifying a noncodeleted tumor, but at the risk of decreasing the specificity and interobserver correlation.

We acknowledge the presence of selection bias because $1 \mathrm{p} / 19 \mathrm{q}$ testing was not performed on all intracranial gliomas during this period, which was largely before the update to the WHO criteria. Because $1 \mathrm{p} / 19 \mathrm{q}$ testing was generally performed on the basis of an oligodendroglial component on standard histologic assessment (usually an oligoastrocytoma), one may have expected a relatively large proportion of $1 \mathrm{p} / 19 \mathrm{q}$ codeleted tumors, but the proportion of $1 \mathrm{p} / 19 \mathrm{q}$ codeleted tumors in our cohort was similar to that in a large series described recently. ${ }^{15}$ Presumably this similarity reflects a balance between the relatively small number of histologic astrocytomas and oligodendrogliomas in our cohort, which were not thought to require $1 \mathrm{p} 19 \mathrm{q}$ testing at the time. Also related to this selection bias, there was only 1 protoplasmic astrocytoma in our cohort (diagnosed before the recent WHO classification). Nevertheless, the substantial number of patients in our cohort with $>50 \%$ T2-FLAIR mismatch suggests that this MR imaging feature is not specific to tumors labeled protoplasmic astrocytomas. This may be because a protoplasmic (or microcystic) appearance on histology occurs on a continuum, and the diagnosis was not clearly defined in the previous WHO classification, ${ }^{22}$ a reason for this entity no longer being recognized in the more recent classification. ${ }^{1}$ Whatever the histologic correlate for T2-FLAIR mismatch, however, it is a very useful biomarker.

More definitive IDH mutation testing was not routinely available for patients with immunohistochemistry negative for IDH1, but the addition of ATRX and TP53 immunohistochemistry data overcomes some of this limitation, suggesting some tumors that are likely to harbor non- $132 \mathrm{H} \mathrm{IDH} \mathrm{muta-}$ tions. Our results thus somewhat support the findings of Patel et $\mathrm{al}^{3}$ that substantial T2-FLAIR mismatch is specific to IDHmutant astrocytomas rather than $I D H$ wild-type astrocytomas, but we are unable to support this definitively. Of note, the single $1 \mathrm{p} 19 \mathrm{q}$ codeleted tumor with immunohistochemistry negative for IDH1 did not demonstrate calcifications or $>50 \%$ T2-FLAIR mismatch; thus, the uncertainty regarding the exact diagnosis according to the updated WHO criteria does not affect the results.

The single-institution nature of our study is a limitation, though in this context, ours is a large cohort, with the number of codeleted tumors demonstrating $>50 \%$ T2-FLAIR mismatch being like that reported for the TCGA/TCIA cohort. ${ }^{3}$ Our study is retrospective, and prospective validation of our results in a different cohort would be important. The targeted nature of our MR imaging assessment is also a potential limitation. We thought that it was important to focus on a small number of MR imaging features, to minimize the chance of finding an association by chance alone. Given the small size of the initial cohort, consisting of only 10 patients, it is possible that some MR imaging features that would have been useful in this context were not identified. In addition, the incidence of calcification is likely to be underestimated in our cohort. First, CTs were not available for all patients, and in patients with only a postoperative CT available, there is the potential for the component containing calcifications to have been resected (and thus not accurately identifiable on the imaging available). Second, susceptibility-weighted imaging was variably available; thus, often calcification could not be accurately differentiated from hemorrhage, in which case tumors were labeled as negative for calcification. We suspect, therefore, that our results underestimate the frequency of calcifications, and the ability of MR imaging to identify codeleted tumors may have been higher if $\mathrm{CT}$ and/or susceptibility-weighted imaging had been performed in all patients. This addition has the potential to further increase the value of the method we have outlined.

\section{CONCLUSIONS}

Some MR imaging features can predict $1 \mathrm{p} / 19 \mathrm{q}$ status with high specificity. In particular, $>50 \%$ T2-FLAIR mismatch is highly predictive of a noncodeleted tumor, while calcifications suggest a $1 \mathrm{p} / 19 \mathrm{q}$ codeleted tumor. Both features have high interobserver correlation. If formal $1 \mathrm{p} / 19 \mathrm{q}$ testing is not possible, these MR imaging features are likely to be more specific for determining $1 p / 19 q$ status than the histologic phenotype and are particularly useful in phenotypic oligoastrocytomas. We thus suggest the use of combined MR imaging-histologic assessment in such situations to optimize diagnosis.

AJNR Am J Neuroradiol 39:687-92 Apr 2018 www.ajnr.org 


\section{REFERENCES}

1. Louis DN, Perry A, Reifenberger G, et al. The $\mathbf{2 0 1 6}$ World Health Organization Classification of Tumors of the Central Nervous System: a summary. Acta Neuropathol 2016;131:803-20 CrossRef Medline

2. Lasocki A, Tsui A, Gaillard F, et al. Reliability of noncontrast-enhancing tumor as a biomarker of IDH1 mutation status in glioblastoma. J Clin Neurosci 2017;39:170-75 CrossRef Medline

3. Patel SH, Poisson LM, Brat DJ, et al. T2-FLAIR mismatch, an imaging biomarker for IDH and 1p/19q status in lower-grade gliomas: a TCGA/TCIA project. Clin Cancer Res 2017;23:6078-85 CrossRef Medline

4. Tay K, Tsui A, Phal P, et al. MR imaging characteristics of protoplasmic astrocytomas. Neuroradiology 2011;53:405-11 CrossRef Medline

5. Saito T, Muragaki Y, Maruyama T, et al. Calcification on CT is a simple and valuable preoperative indicator of $1 \mathrm{p} / 19 \mathrm{q}$ loss of heterozygosity in supratentorial brain tumors that are suspected grade II and III gliomas. Brain Tumor Pathol 2016;33:175-82 CrossRef Medline

6. Johnson DR, Diehn FE, Giannini C, et al. Genetically defined oligodendroglioma is characterized by indistinct tumor borders at MRI. AJNR Am J Neuroradiol 2017;38:678-84 CrossRef Medline

7. Smits M. Imaging of oligodendroglioma. Br J Radiol 2016;89: 20150857 CrossRef Medline

8. Osborne AG. Osborne's Brain: Imaging, Pathology and Anatomy. Salt Lake City: Amirsys; 2013:496

9. Law M, Brodsky JE, Babb J, et al. High cerebral blood volume in human gliomas predicts deletion of chromosome 1p: preliminary results of molecular studies in gliomas with elevated perfusion. $J$ Magn Reson Imaging 2007;25:1113-19 CrossRef Medline

10. Law $\mathrm{M}$, Yang $\mathrm{S}$, Wang $\mathrm{H}$, et al. Glioma grading: sensitivity, specificity, and predictive values of perfusion MR imaging and proton MR spectroscopic imaging compared with conventional MR imaging. AJNR Am J Neuroradiol 2003;24:1989-98 Medline

11. Lasocki A, Tsui A, Tacey MA, et al. MRI grading versus histology: predicting survival of World Health Organization grade II-IV astrocytomas. AJNR Am J Neuroradiol 2015;36:77-83 CrossRef Medline
12. Gonzales M, Dale S, Susman M, et al. Quantitation of chromosome $1 \mathrm{p}$ and $19 \mathrm{q}$ deletions in glial tumours by interphase FISH on formalin-fixed paraffin-embedded tissue. J Clin Neurosci 2006;13:96-101 CrossRef Medline

13. VASARI Research Project. https://wiki.cancerimagingarchive.net/ display/Public/VASARI+Research+Project. Updated March 25, 2015. Accessed August 20, 2015

14. Louis DN, Ohgaki H, Wiestler OD, et al. World Health Organization Histological Classification of Tumours of the Central Nervous System. Lyon: International Agency for Research on Cancer; 2016

15. Brat DJ, Verhaak RG, Aldape KD, et al; Cancer Genome Atlas Research Network. Comprehensive, integrative genomic analysis of diffuse lower-grade gliomas. $N$ Engl J Med 2015;372:2481-98 CrossRef Medline

16. Clark KH, Villano JL, Nikiforova MN, et al. 1p/19q testing has no significance in the workup of glioblastomas. Neuropathol Appl Neurobiol 2013;39:706-17 CrossRef Medline

17. Ito M, Wakabayashi $\mathrm{T}$, Natsume A, et al. Genetically heterogeneous glioblastoma recurring with disappearance of $1 p / 19 q$ losses: case report. Neurosurgery 2007;61:E168-69; discussion E169 CrossRef Medline

18. Huse JT, Diamond EL, Wang L, et al. Mixed glioma with molecular features of composite oligodendroglioma and astrocytoma: a true “oligoastrocytoma"? Acta Neuropathol 2015;129:151-53 CrossRef Medline

19. Qu M, Olofsson T, Sigurdardottir S, et al. Genetically distinct astrocytic and oligodendroglial components in oligoastrocytomas. Acta Neuropathol 2007;113:129-36 CrossRef Medline

20. Lasocki A, Gaillard F, Tacey M, et al. Incidence and prognostic significance of non-enhancing cortical signal abnormality in glioblastoma. J Med Imaging Radiat Oncol 2016;60:66-73 CrossRef Medline

21. Tang YM, Ngai S, Stuckey S. The solitary enhancing cerebral lesion: can FLAIR aid the differentiation between glioma and metastasis? AJNR Am J Neuroradiol 2006;27:609-11 Medline

22. Louis DN, Ohgaki H, Wiestler OD, et al. World Health Organization Histological Classification of Tumours of the Central Nervous System. Lyon: International Agency for Research on Cancer; 2007 